\title{
Long non-coding RNA MALAT1 correlates with cell viability and mobility by targeting miR-22-3p in renal cell carcinoma via the PI3K/Akt pathway
}

\author{
ZHONG $\mathrm{LI}^{1}$, ZHIQIANG MA ${ }^{2}$ and XIANGDONG $\mathrm{XU}^{3}$ \\ ${ }^{1}$ Department of Urology, The Second Hospital Affiliated to Tianjin Medical University, Tianjin 300211; \\ ${ }^{2}$ Department of Urology, The Third Hospital of Shijiazhuang, Shijiazhuang, Hebei 050000; \\ ${ }^{3}$ Department of Urology, Tai'an City Central Hospital, Tai'an, Shandong 271000, P.R. China
}

Received September 29, 2017; Accepted May 9, 2018

DOI: 10.3892/or.2018.6853

\begin{abstract}
Renal cell carcinoma (RCC) is one of the most common types of cancer of the urinary tract in the world. Long non-coding RNA MALAT1 (lncR-MALAT1) is upregulated in RCC and is associated with the proliferation and migration of RCC. The present study aimed to investigate the regulating role of lncR-MALAT1 in RCC as well as the possible underlying mechanisms. The relative expression of MALAT1 and miR-22-3p in RCC tumor tissues and cell lines was detected by qRT-PCR. CCK-8 and wound healing assay were used to evaluate cell proliferation and migration ability. Western blot analysis was used to detect the expression of $\mathrm{Ki}-67$, proliferating cell nuclear antigen (PCNA), matrix metalloproteinase-3 (MMP-3), migration and invasion inhibitory protein (MIIP), p-PI3K and p-Akt. The relationship between MALAT1 and miR-22-3p was examined by bioinformatic prediction analysis and luciferase reporter assay. Immunofluorescence was used to detect the activation of Akt. MALAT1 was highly expressed and the expression of miR-22-3p was suppressed in RCC tissues and cell lines. ShRNA-mediated knockdown of MALAT1 significantly inhibited the viability and mobility of RCC cells in vitro and in vivo. Further experiments revealed that miR-22-3p was a target of MALAT1 and that miR-22-3p inhibitor abolished the effect of MALAT1 shRNA
\end{abstract}

Correspondence to: Dr Xiangdong Xu, Department of Urology, Tai'an City Central Hospital, 29 Longtan Road, Taishan, Tai'an, Shandong 271000, P.R. China

E-mail: xuxiangdongsdta@sina.com

Abbreviations: MALAT1, metastasis-associated lung adenocarcinoma transcript 1; lncRNA, long non-coding RNA; RCC, renal cell carcinoma; miRNA, microRNA; MMP-3, matrix metaloproteinase-3; PCNA, proliferating cell nuclear antigen; MIIP, migration and invasion inhibitory protein; FBS, fetal bovine serum

Key words: long non-coding RNA MALAT1, viability, mobility, miR-22-3p, renal cell carcinoma, PI3K/Akt pathway on cell proliferation, migration and inactivation of PI3K/AKT pathway. In conclusion, IncR-MALAT1 affected the proliferation and migration of RCC cells by targeting miR-22-3p through the inactivation of the PI3K/Akt signaling pathway.

\section{Introduction}

Renal cell carcinoma (RCC) is one of the most common cancers of the urinary tract in the world, accounting for 2-3\% of adult cancers (1). It is reported that there are 209,000 new cases of RCC annually in the world and the mortality rate is as high as $50 \%$ (2). The treatment effects of conventional radiotherapy and chemotherapy are not satisfactory and the treatment of RCC is mainly based on surgery (3). Therefore, it is essential to obtain a better understanding of the RCC development mechanism and find new therapeutic targets to reduce RCC mortality rate.

Human gene sequencing results have revealed that $>90 \%$ of human DNA sequences is involved in transcription, but only $2 \%$ has protein encoding functions. RNA that does not have a protein coding function is categorized as non-coding RNA (4). Non-coding RNA is divided into long-chain non-coding RNA (lncRNA) and short-chain non-coding RNA according to the size of RNA $(4,5)$. Among them, short-chain non-coding RNA, such as microRNA (miRNA), plays an important role in regulating gene expression and cell function (6). Previous research has revealed that miRNA-22 (miR-22) is lowly expressed in breast cancer, esophageal squamous cell carcinoma, gastric and colorectal cancer. miR-22 inhibited cell growth, migration and epithelial-mesenchymal transition in the above-mentioned cancers (7-11). It was reported that miR-22 inhibited the invasion and migration of gastric cancer cells by downregulating the expression of MMP-14 and Snail (12). In addition, it has been reported that miR-22 inhibited the proliferation and migration of clear RCC cells by regulating the expression of PTEN, indicating that miRNA-22 has an inhibitory effect in RCC (13).

Recent research has revealed that lncRNA has a variety of biological functions in cancer progression $(14,15)$. IncRNAs, such as HOTAIR, TUG1, SPRY4-IT and metastasis-associated lung adenocarcinoma transcript 1 lncRNA (lncR-MALAT1) act as important regulators in the proliferation and metastasis 
of cancer cells (16-18). IncR-MALAT1 is an IncRNA which is located on the 11q13.1 chromosome (19). Previous research has demonstrated that MALAT1 is highly expressed in a variety of cancers, such as lung, breast, liver, pancreatic and colon cancer (20). Hirata et al (19) revealed that MALAT1 promoted the mobility of RCC cells by influencing the interaction between Ezh2 and miR-205. Furthermore, MALAT1 was reported to affect the growth of melanoma by targeting miR-22 (21). However, whether miR-22 is a target of MALAT1 in the proliferation and migration of RCC cells has not been reported.

In the present study, we identified that the expression of MALAT1 was elevated, and the expression of miR-22-3p was markedly decreased in RCC tumor tissues and cell lines. In addition, silencing MALAT1 obviously inhibited the proliferation and migration of tumor cells in vitro and in vivo. In addition, miR-22-3p was demonstrated to be a target of MALAT1. miR-22-3p inhibitor reversed the inhibitory effect of shRNA-MALAT1 on the progression of RCC and the PI3K/Akt signaling pathway. Collectively, MALAT1 affected the development of RCC by targeting miR-22-3p via the PI3K/Akt signaling pathway.

\section{Materials and methods}

Sample collection. Thirty pairs of RCC specimens and adjacent normal tissues were obtained from patients who underwent surgical treatment from October 2015 to August 2016 in Tai'an City Central Hospital. Written informed consent was obtained prior to resection from patients and the study was approved by the Ethics Committee of Tai'an City Central Hospital.

Cell lines. RCC cell lines 786-O, Caki-1, Ketr-3, RT112 and T24, as well as the normal renal epithelial cells Ect1/E6E7 were purchased from the American Type Culture Collection (ATCC, Gaithersburg MD, USA). The 786-O, Ketr-3, RT-112 and T24 cells were cultured in RPMI-1640 medium (Gibco, Rockville, MD, USA) containing $10 \%$ fetal bovine serum (FBS; Gibco). The Caki-1 cells were cultured in McCoy's 5A medium containing 10\% FBS, and Ect1/E6E7 cells were cultured in EMEM medium (Gibco) containing 10\% FBS. All cells were cultured in incubators at $37^{\circ} \mathrm{C}$ in a $5 \% \mathrm{CO}_{2}$ atmosphere.

Quantitative real-time polymerase chain reaction ( $q R T-P C R$ ). Total RNA was extracted from cells and tissues using the TRIzol kit (Invitrogen; Thermo Fisher Scientific, Inc., Waltham, MA, USA) according to the manufacturer's instructions and cDNA was synthesized using Reverse Transcription kit (Thermo Fisher Scientific). Quantitative analysis of cDNA (Thermo Fisher Scientific) was performed using a Fluorescence Quantitative PCR kit (Sangon Biotech Co., Ltd. Shanghai, China). The amplification primers used were as follows: MALAT1 sense, 5'-CTCACTAAAGGCACCGAA GG-3' and antisense, 5'-GGCAGAGAAGTTGCTTGTGG-3'; miR-22-3p sense, 5'-AAGCTGCCAGTTGAAGAACTGTA-3' and antisense, 5'-CTCGCTTCGGCAGCACA-3'.

Cell transfection. Caki-1 cells were seeded in 24-well plates at $1 \times 10^{5}$ cells/well and were transfected with specified fragments for $24 \mathrm{~h}$, when the cell aggregation rate was over $80 \%$. Mimics/inhibitors specific for miR-22-3p and short hairpin
RNA (shRNA)/scramble fragments targeting MALAT1 were designed and purchased from Hanheng Biotechnology Co., Ltd. (Shanghai, China). Recombinant lentiviral vector for knocking out MALAT1 was constructed and named LV-MALAT1 shRNA and the control group was named LV-MALAT1 scramble group.

Cell viability. Caki-1 cells were transfected with shRNAMALAT1 or MALAT1 scramble, and cell viability was evaluated according to the instructions of the CCK-8 assay kit (Sigma-Aldrich, St. Louis, MO, USA).

Wound healing assay. Five parallel lines were evenly drawn on the back of the 6-well plate using marker pens and the Caki-1 cells were grown in 6-well plates at a concentration of $2 \times 10^{5}$ cells $/ \mathrm{ml}$. After incubation for $24 \mathrm{~h}$, the tip was scratched perpendicularly to the horizontal line on the back of the 6-well plate. Destroyed cells were rinsed off with phosphate-buffered saline (PBS) and cultured in serum-free medium for another $24 \mathrm{~h}$. Cell migration was observed and imaged at 0 and $24 \mathrm{~h}$ with a digital camera (Olympus Corp., Tokyo, Japan).

Western blot analysis. Protein samples were extracted from cells and tissues with protein lysate, and the protein concentration was assessed using a BCA kit (Pierce, Rockford, IL, USA). The protein was separated by SDS-PAGE and the isolated protein was transferred to the polyvinylidene fluoride (PVDF) membrane by semi-dry method and then was blocked in 5\% skim milk at room temperature, for $2 \mathrm{~h}$. The membrane was incubated with the primary antibodies (anti-Ki-67, cat. no. ab16667; anti-PCNA, cat. no. ab29; anti-MMP3, cat. no. ab53015; anti-MIIP, cat. no. ab167197; p-PI3K, cat. no. ab182651; anti-p-AKT, cat. no. ab131443; anti-AKT, cat. no. ab179463; all at the dilution 1:1,000) and the corresponding HRP-conjugated secondary antibodies (cat. nos. ab6728 and ab6721 at the dilution 1:5,000; obtained from Abcam, Cambridge, UK) and was extensively washed several times with PBST. The proteins were detected using a ChemiDoc XRS imaging system and were analysed through Quantity One analysis software (Bio-Rad Laboratories, San Francisco, CA, USA). GAPDH was used as the internal control.

Luciferase activity assays. The binding site of MALAT1 on miR-22-3p was predicted through bioinformatic analysis (TargetScan, http: //www.targetscan.org/). PCR amplification of MALAT1 fragments contained a fragment of the miR-22-3p binding site, which was inserted into the pMIR-REPORT vector. The mutant plasmid was used as the control group. Caki-1 cells were co-transfected with MALAT1 WT/MALAT1-MUT and/or miR-22-3p mimic for $24 \mathrm{~h}$. Fluorescence intensity was evaluated according to the Dual-Luciferase Reporter kit (Promega Corporation, Madison, WI, USA).

Immunofluorescence. Caki-1 cells were treated with $4 \%$ paraformaldehyde for $30 \mathrm{~min}$ and $0.5 \%$ Triton X-100 for $30 \mathrm{~min}$ and then were blocked with $5 \%$ goat serum for $2 \mathrm{~h}$ at room temperature. The cells were then incubated with the primary antibody (Akt, 1:200; cat. no. ab179463; Abcam) overnight at $4^{\circ} \mathrm{C}$. On the second day, the cells were incubated with the corresponding FITC-conjugated secondary antibodies 
A
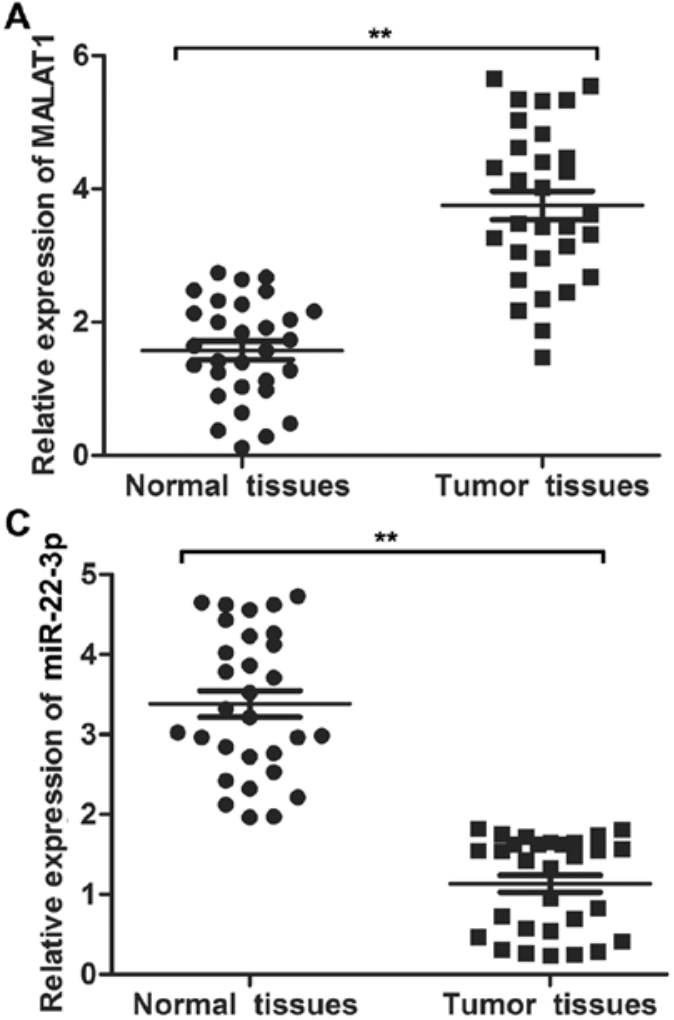

B

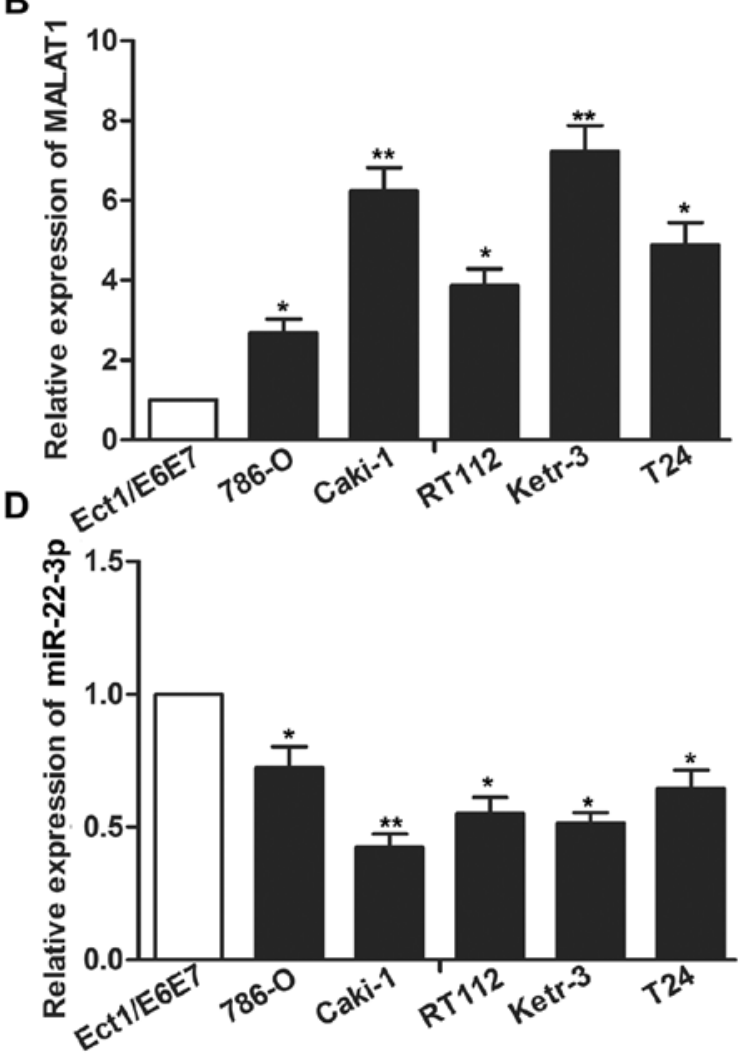

Figure 1. The expression of MALAT1 is increased in RCC tumor tissue and cell lines. (A) qRT-PCR was used to detect the expression of MALATI in RCC tissues and normal adjacent tissues ( $\mathrm{n}=30$ ). (B) qRT-PCR was used to detect the expression of MALAT1 in RCC cell lines and normal renal epithelial cells. (C) qRT-PCR was used to detect the expression of miR-22-3p in RCC tissues and normal adjacent tissues. (D) qRT-PCR was used to detect the expression of miR-22-3p in RCC cell lines and normal renal epithelial cells. The adjacent tissues and normal renal epithelial cells were used as normal control. All experiments were repeated at least three times. ${ }^{*} \mathrm{P}<0.05,{ }^{* *} \mathrm{P}<0.01$ vs. the control group.

(1:2,000; cat. no. ab6785; Abcam) in the dark for 50 min, and then were stained with DAPI. Cell fluorescence was observed under a fluorescence microscope (Olympus Corp.).

RCC xenografts. Sixteen male specific-pathogen-free (SPF) nude mice $(\sim 20 \mathrm{~g})$ were purchased from the Beijing Experimental Animal Center (Beijing, China). The animal experiments were performed according to the guidelines of the National Institute of Health (NIH). All animal experiments were approved by the Medical Ethics Committee of The Second Hospital Affiliated to Tianjin Medical University (Tianjin, China). An RCC xenograft mouse model was created by subcutaneous injection of $1 \times 10^{7}$ Caki-1 cells transfected with LV-MALAT1 shRNA or LV-MALAT1 scramble to SPF nude mice. When sacrificed, the weight of mice was $\sim 25 \mathrm{~g}$. The tumor volume was evaluated every 5 days until 30 days after the development of a palpable tumor according to the formula $\left(a \times b^{2}\right) / 2$, where $a$ and $b$ are the largest diameter and the perpendicular diameter, respectively.

Statistical analysis. All data were analyzed using the SPSS 19.0 software (IBM Corp., Armonk, NY, USA) and the results were expressed as the mean \pm standard deviation (SD). The Student's t-test was used to assess comparisons between two groups. Multiple-group comparisons were made using one-way analysis of variance (ANOVA). $\mathrm{P}<0.05$ was considered to indicate a statistically significant difference.

\section{Results}

MALAT1 is upregulated and miR-22-3p is downregulated in the RCC tissues and cell lines. The expression of MALAT1 and miR-22-3p in RCC tissues and cell lines was detected through qRT-PCR. As displayed in Fig. 1A, the expression level of MALAT1 in the RCC tumor tissue was significantly higher than that in adjacent normal tissues $(\mathrm{P}<0.01)$. Concurrently, the expression of MALAT1 in the RCC cell lines was significantly increased compared with the normal renal epithelial cells Ect1/E6E7 (Fig. 1B; $\mathrm{P}<0.05$ and $\mathrm{P}<0.01$ ). In addition, the expression level of miR-22-3p in the RCC tumor tissues and cell lines was significantly decreased compared with the control tissues and cell line (Fig. 1C and D; P $<0.05$ and $\mathrm{P}<0.01)$. The aberrant expression of MALAT1 and miR-22-3p compared with normal renal epithelial cells indicated a systematic predictable link between MALAT1/miR-22-3p and the progression of RCC.

Effect of MALAT1 in RCC cell proliferation and metastasis. Previous research has demonstrated that MALAT1 regulates the proliferation and migration of a variety of cancer cells. In the present study, we selected the Caki-1 cell line for the following experiments as it has the relatively highest expression of MALAT1 and the lowest expression level of miR-22-3p in the related cell lines. The expression of MALAT1 was significantly downregulated through transfection with MALAT1 

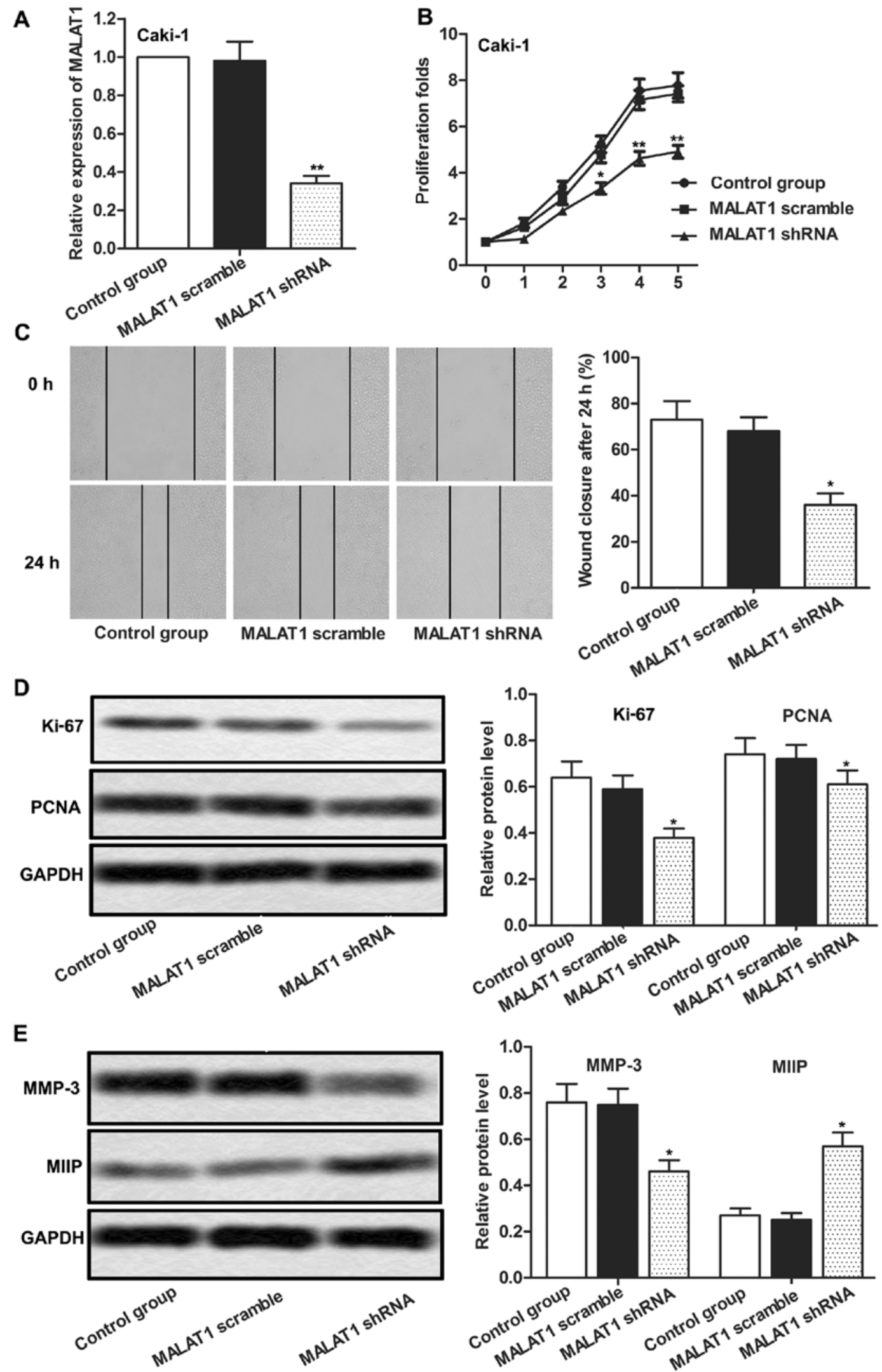

Figure 2. MALAT1 promotes RCC cell proliferation and migration. The Caki-1 cells were transfected with MALAT1 shRNA or MALAT1 scramble or left untreated as the control. (A) qRT-PCR was used to detect the expression of MALATI. (B) CCK-8 was used to detect cell viability. (C) Wound healing assay was used to detect cell migration. (D and E) Western blot analysis was used to detect the expression of Ki-67, PCNA, MMP-3 and MIIP. GAPDH was used as internal reference, ${ }^{*} \mathrm{P}<0.05,{ }^{* *} \mathrm{P}<0.01$ vs. control group.

shRNA in Caki-1 cells compared with the scramble group and the control group (Fig. 2A; $<<0.01$ ). Transfection with
MALAT1 shRNA suppressed cell proliferation compared with MALTA1 scramble group (Fig. 2B; $\mathrm{P}<0.05$ and $\mathrm{P}<0.01$ ). 
A

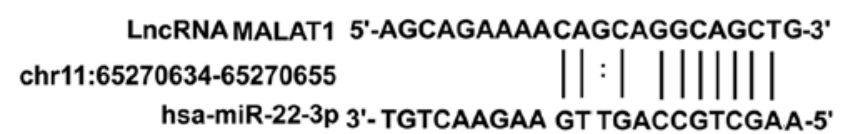

B

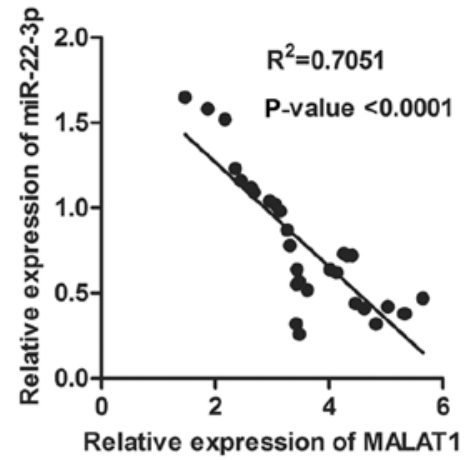

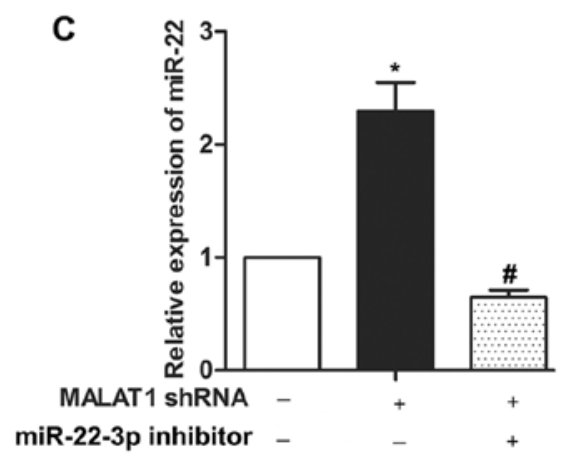

F

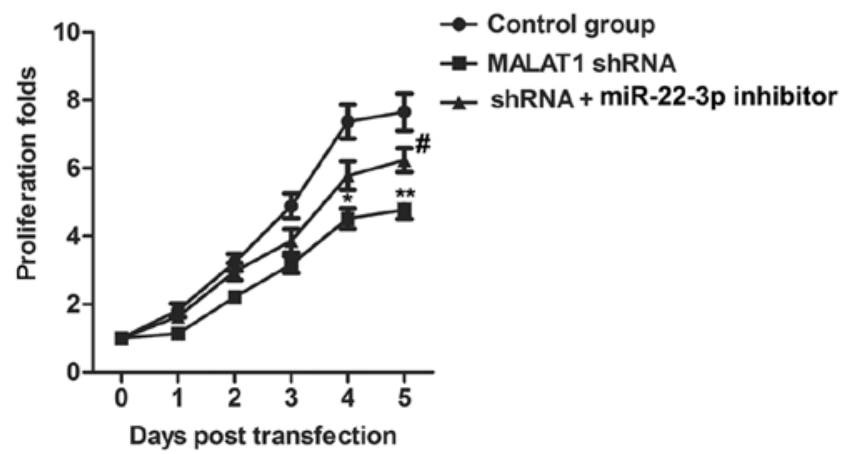

D

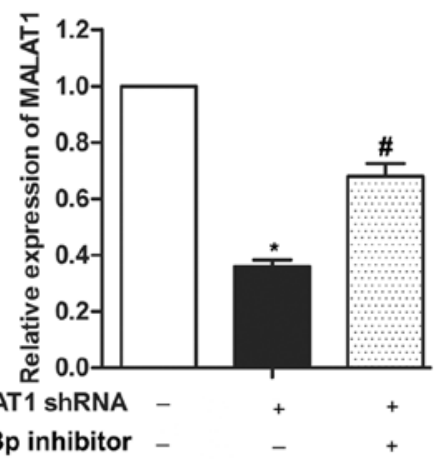

E

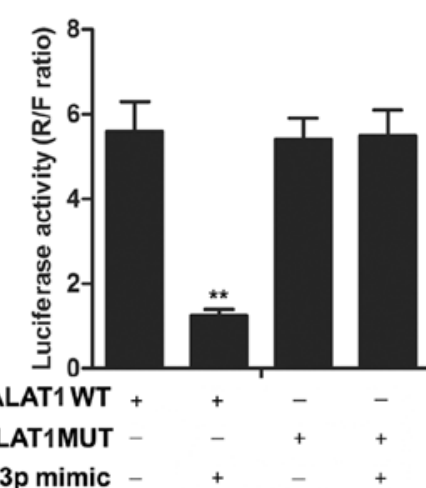
miR-22-3p mimic

\author{
G
}

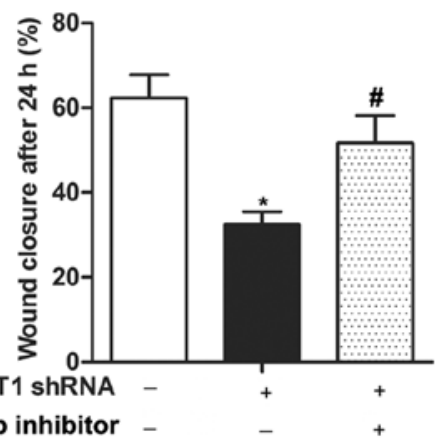

Figure 3. MALAT1 promotes the proliferation and migration of RCC cells by targeting miR-22-3p. (A) Bioinformatic analysis was used to predict the targeting relationship between MALATI and miR-22-3p. (B) Analysis of the correlation between MALAT1 and the expression of miR-22-3p in RCC tissues. (C) The cells were transfected with MALAT1 shRNA or co-transfected with miR-22-3p inhibitor. qRT-PCR was used to detect the effect of MALAT1 shRNA on miR-22-3p expression. (D) qRT-PCR was used to detect the effect of MALAT1 shRNA and miR-22-3p inhibitor on the expression of MALAT1. (E) Luciferase reporter assay further identified the targeting relationship between MALATI and miR-22-3p. (F) Analysis results of the CCK-8 assay which was used to detect cell proliferation. (G) Analysis results of the wound healing assay which was used to evaluate cell migration ability. All experiments were repeated at least three times. ${ }^{*} \mathrm{P}<0.05,{ }^{* *} \mathrm{P}<0.01$ vs. the control group, ${ }^{\#} \mathrm{P}<0.05$ vs. MALAT1 shRNA group.

Furthermore, the wound healing assay exhibited that the migration ability of cells in the MALAT1 shRNA group was much lower than that of the control group (Fig. $2 \mathrm{C} ; \mathrm{P}<0.05$ ). The results of the western blot analysis revealed that MALAT1 shRNA significantly suppressed the expression of Ki-67 and proliferating cell nuclear antigen (PCNA) compared with the scramble and the control group $(\mathrm{P}<0.05)$ (Fig. 2D). As expected, MALAT1 shRNA significantly inhibited the expression of matrix metalloproteinase-3 (MMP-3) and promoted the expression of migration and invasion inhibitory protein (MIIP) (Fig. 2E; P<0.05). The above-mentioned results indicated that MALAT1 shRNA significantly inhibited the proliferation and migration of RCC cells.

MALAT1 regulates cell viability and mobility by targeting $m i R-22-3 p$ in $R C C$. To investigate the related mechanism by which MALAT1 regulates the progression of RCC cells, we found a binding site for MALAT1 on miR-22-3p through bioinformatic analysis (TargetScan, http: //www.targetscan. org/) (Fig. 3A). Concurrently, there were marked negative relevant relations between the expression of MALAT1 and miR-22-3p in RCC tissues (Fig. 3B). As displayed in Fig. 3C, MALAT1 shRNA significantly elevated the expression of miR-22-3p in RCC cells $(\mathrm{P}<0.05)$, but then this effect was reversed through the co-transfection with miR-22-3p inhibitor $(\mathrm{P}<0.05)$. Furthermore, the inhibiting effect of MALAT1 shRNA on the expression of MALAT1 was partially reversed by the co-transfection of miR-22-3p inhibitor, identifying that miR-22-3p inhibitor elevated the level of MALAT1 in turn (Fig. 3D; $\mathrm{P}<0.05$ and $\mathrm{P}<0.05$, respectively). Subsequently, we used the luciferase reporter assay to further examine the targeting relationship between MALAT1 and miR-22-3p. The results demonstrated that the luciferase signal in the lncR-MALAT1 MUT and the lncR-MALAT1 WT group 
A

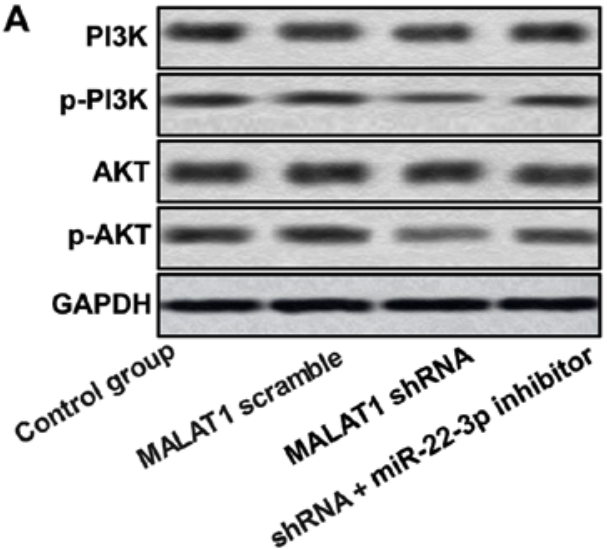

B
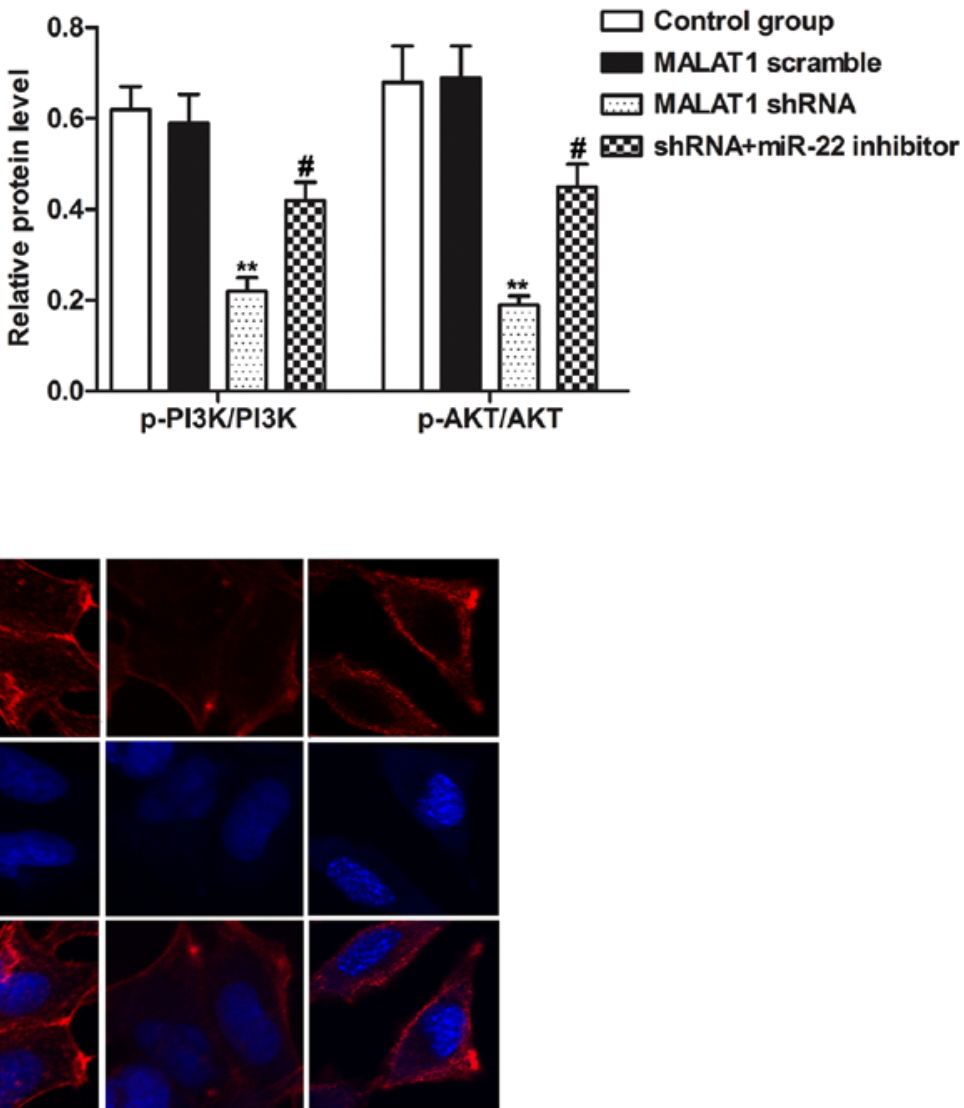
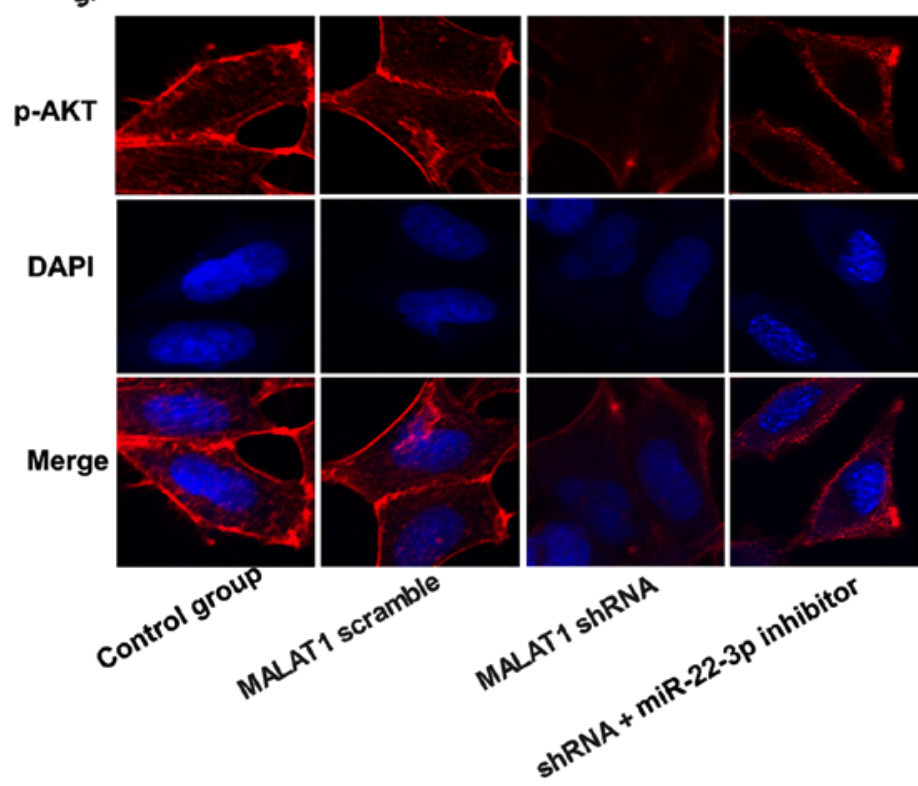

Figure 4. MALAT1 shRNA inhibits the PI3K/Akt pathway activation. The cells were divided into the control group, the MALAT1 scramble group, the MALAT1 shRNA group and the shRNA + miR-22-3p inhibitor group. (A) Western blot analysis was used to detect the expression of p-PI3K and p-Akt in RCC cells. (B) Immunofluorescence was used to detect the distribution of Akt expression. GAPDH was used as an internal control. ${ }^{* *} \mathrm{P}<0.01$ vs. the control group, ${ }^{\#} \mathrm{P}<0.05$ vs. MALAT1 shRNA group.

remained at the same level, but the addition of miR-22-3p mimic significantly inhibited the luciferase activity by binding to MALAT1 WT fragments. However, the regulatory relationship disappeared when binding to the MALAT1 MUT fragments, indicating that there existed a targeting relationship between MALAT1 and miR-22-3p (Fig. 3E; P<0.01). Furthermore, miR-22-3p inhibitor significantly reversed the inhibitory effect of MALAT1 shRNA on the proliferation and migration of Caki-1 cells (Fig. 3F and G; $\mathrm{P}<0.05, \mathrm{P}<0.01$ and $\mathrm{P}<0.05$ ). These results indicated that MALAT1 may promote the proliferation and migration of RCC cells by targeting miR-22-3p.

MALAT1 shRNA restrains the activation of the PI3K/AKT pathway. Recent research has indicated that PI3K/Akt signaling pathway is closely related to the proliferation and migration of cancer cells $(24,25)$. We have also demonstrated that MALAT1 shRNA inhibited the proliferation and migration of Caki-1 cells in the above-mentioned experiments. Therefore, we further investigated the effect of MALAT1 shRNA on the PI3K/Akt pathway. As displayed in Fig. 4A, MALAT1 shRNA significantly inhibited the expression of p-PI3K and p-Akt, but the addition of miR-22-3p inhibitor into the MALAT1
shRNA-treated Caki-1 cells significantly reversed the inhibitory effect of MALAT1 shRNA on the expression of p-PI3K and $\mathrm{p}$-Akt $(\mathrm{P}<0.01, \mathrm{P}<0.05)$. In addition, the expression of Akt in the MALAT1 shRNA group was much lower than that in the control group, indicating that MALAT1 shRNA inhibited the activation of Akt (Fig. 4B). Concurrently, miR-22-3p inhibitor reversed the inhibitory effect of MALAT1 shRNA on Akt accumulation on the cell membrane (Fig. 4B). All these results revealed that MALAT1 shRNA inhibited the activation of PI3K/Akt signaling pathway through the upregulation of the expression of miR-22-3p.

MALATI shRNA suppresses tumor growth and metastasis in vivo. To further validate the above results in vivo, we replicated the xenograft animal model of RCC and explored the effect of MALAT1 shRNA on tumor growth and metastasis. The results demonstrated that MALAT1 shRNA inhibited the growth of RCC tumor and elevated the expression of miR-22-3p in vivo (Fig. $5 \mathrm{~A}$ and $\mathrm{B} ; \mathrm{P}<0.05, \mathrm{P}<0.01$ ). Concurrently, the expression of PCNA and MMP-3 in the shRNA-MALAT1 group was significantly lower compared with the control group. The ratio of p-Akt/Akt was also significantly lower than 
A

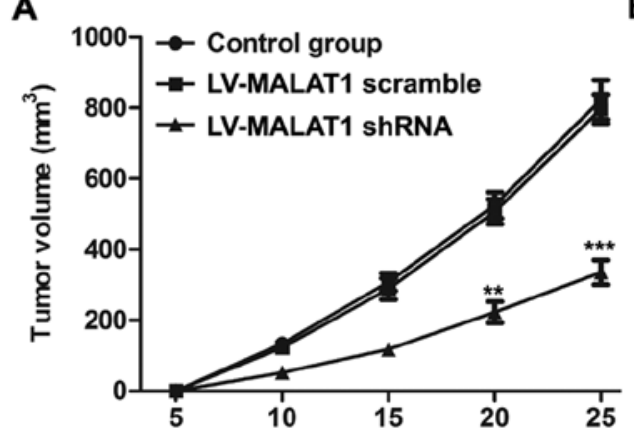

C

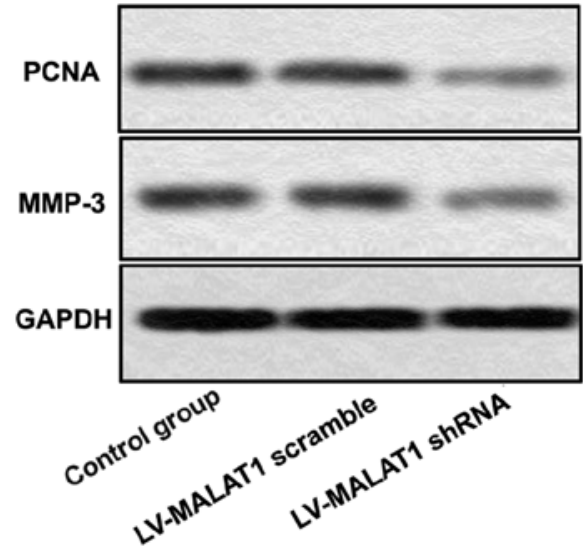

D

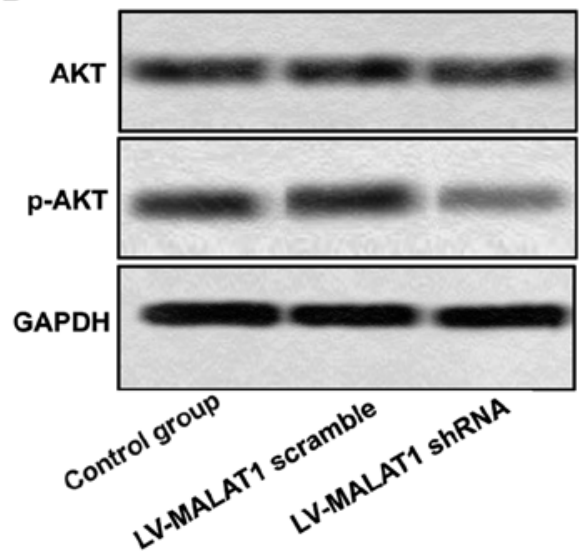

B
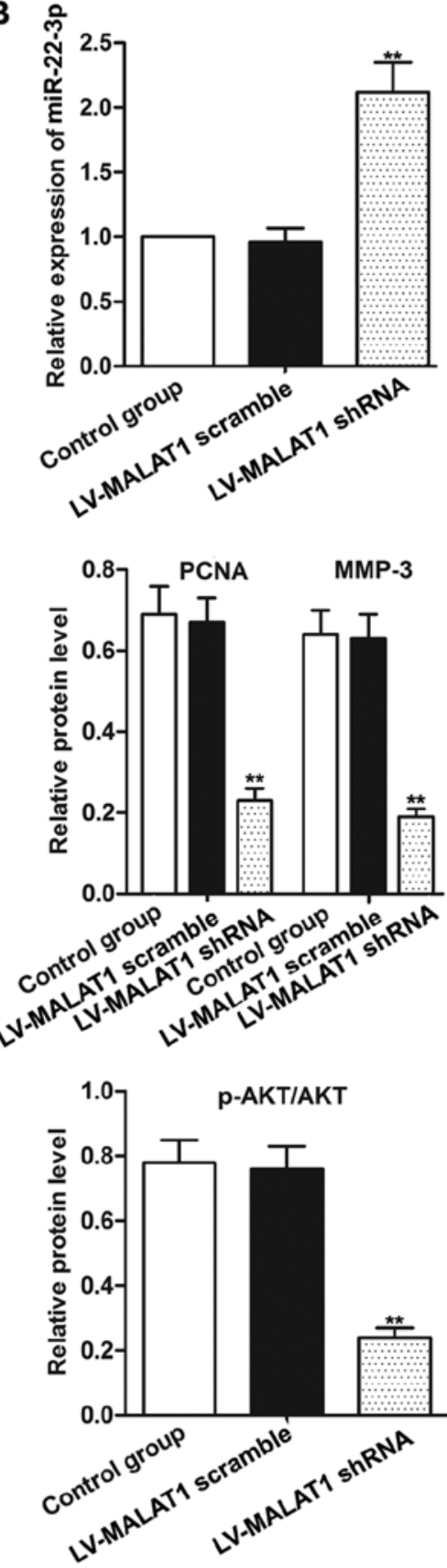

Figure 5. MALAT1 shRNA inhibits RCC tumor growth and metastasis. Caki-1 cells were pre-treated with LV-MALAT1 shRNA or LV-MALAT1 scramble or left untreated. RCC xenograft mouse model was created by subcutaneous injection of recombinant cell lines to SPF nude mice. (A) Tumor volume growth curve. (B) qRT-PCR was used to detect the expression of miR-22-3p in tumor tissue. (C) Western blot analysis was used to detect the expression of PCNA and MMP-3 in tumor tissues. (D) Western blot analysis was used to detect the expression of p-Akt and Akt in tumor tissues. PCNA, MMP-3 and p-Akt/Akt were quantitatively analyzed. GAPDH was used as an internal control. ${ }^{* *} \mathrm{P}<0.01,{ }^{* * *} \mathrm{P}<0.001$ vs. the control group.

that of the control group (Fig. $5 \mathrm{C}$ and $\mathrm{D}, \mathrm{P}<0.01)$. These results further indicated that MALAT1 shRNA can also inhibit the growth and metastasis of RCC tumors by inhibiting the PI3K/Akt signaling pathway activation in vivo.

\section{Discussion}

IncRNA is a class of transcripts with more than 200 nucleotides which do not have protein-coding abilities (4). Recent research has shown that IncRNA plays a pivotal role in the development of many tumors, but the related mechanism remains to be investigated (22). In the present study, we first investigated the interaction of MALAT1 and miR-22-3p in the development of RCC.

Lu et al (23) identified that the overexpression of IncRNA BC032469 promoted the proliferation of gastric cancer cells through the downregulation of miR-1207-5p. A previous study reported that the overexpression of lncRNA ATB in hepatocellular carcinoma competed with miR-200, leading to an upregulation of the expression of ZEB1 and ZEB2, which promoted the metastasis of hepatocellular carcinoma (24). MALAT1 was firstly discovered in small cell lung cancer and 
is often used as a prognostic indicator of lung cancer metastasis (25). Numerous studies have revealed that MALAT1 is closely related to multiple cancers by regulating cell viability and mobility (26). Consistent with previous studies, in the present study, we revealed that MALAT1 was highly expressed in RCC tissues and cell lines. The CCK- 8 and wound healing assay further revealed that the knockdown of MALAT1 successfully inhibited cell proliferation and migration. The result of western blot analysis also demonstrated the inhibiting effect of MALAT1 shRNA on cell proliferation and migration in RCC cell lines by regulating the expression of cell proliferation and migration marker proteins. These results revealed that MALAT1 shRNA suppressed the progression of RCC.

A previous study has demonstrated that MALAT1 is a class of ceRNAs which promotes cancer development by binding to miRNAs (27). As previously reported, MALAT1 binds to miR-1 and inhibits its binding to messenger RNA, thus promoting the invasion and metastasis of breast cancer cells (28). MALAT1 regulates the development of RCC by binding to miR-200 and miR-205 (19,29). Recent research has also revealed that miR-22 functions as a 'double-edged sword' in human cancer (30). The overexpression of miR-22 can promote cancer cell proliferation and inhibit cancer cell apoptosis in prostate cancer and chronic lymphocytic leukemia $(31,32)$. However, the expression of miR-22 is suppressed in gastric cancer, melanoma, glioma, RCC and other types of cancer. Zhang et al (33) found that miR-22 expression in RCC was reduced and it played an inhibiting role in RCC progression. In addition, it has been demonstrated that MALAT1 competed with miR-22-3p for endogenous RNA to protect endothelium from ox-LDL-induced endothelial dysfunction (34). Similarly, suppressed expression of miR-22-3p in RCC tumor tissues and cell lines was detected in the present study. In addition, bioinformatic analysis was used to predict the targeting relationship between MALAT1 and miR-22-3p. The luciferase assay further confirmed the targeting relationship between MALAT1 and miR-22-3p. Concurrently, miR-22-3p inhibitor significantly reversed the inhibitory effect of MALAT1 shRNA on the proliferation and migration of Caki-1 cells. In summary, MALAT1 regulated the proliferation and migration of RCC cells by targeting miR-22-3p.

The PI3K/Akt signaling pathway is an important pathway for regulating cell cycle, proliferation and apoptosis (35). Previous research has shown that miR-22 regulates the activation of PI3K/Akt pathway (32). MALAT1 can promote the proliferation and epithelial-mesenchymal transition of cholangiocarcinoma and breast cancer cells by activating the PI3K/Akt signaling pathway $(36,37)$. Therefore, we considered whether MALAT1 promoted the proliferation of RCC cells via the PI3K/Akt pathway. We found that MALAT1 shRNA (the experiments were conducted using the most effective among three pairs of shRNAs targeting MALAT1) inhibited the phosphorylation of PI3K and p-Akt and membrane metastasis of Akt in RCC cells, thus inhibiting the PI3K/Akt pathway activation. The co-transfection of miR-22-3p significantly reversed the inhibiting effect of MALAT1 shRNA on the PI3K/Akt pathway. These results indicated that MALAT1 shRNA suppressed the activation of the PI3K/Akt signaling pathway by targeting miR-22-3p.

Following the observation that MALAT1 shRNA regulated cell proliferation and migration in RCC cell lines, the effect of MALAT1 shRNA in RCC tumor was further investigated.
As previously described, MALAT1 promoted tumor growth and metastasis by inducing epithelial-mesenchymal transition in oral squamous cell carcinoma (38). A previous study has also demonstrated that MALAT1 promoted tumor growth and metastasis in colorectal cancer through binding to SFPQ (39). Similarly, in the present study, MALAT1 shRNA was found to suppress tumor growth in an RCC xenograft animal model. Furthermore, MALAT1 shRNA elevated the expression of miR-22-3p and inactivated the PI3K/Akt signaling pathway in vivo. The present study revealed that MALAT1 shRNA suppressed RCC progression in vivo.

In summary, the present study demonstrated that the expression of lncR-MALAT1 was upregulated in RCC tissues and cell lines. Cell proliferation and motility was suppressed by knocking out the expression of MALAT1 by MALAT1 shRNA in RCC cell lines. Further research revealed that miR-22-3p was a direct target of MALAT1 and miR-22-3p inhibitor counteracted the effect of MALAT1 shRNA on cell viability and motility. Furthermore, we found that MALAT1 exerted its regulatory role by inactivating the PI3K/Akt signaling pathway. The in vivo experiments further demonstrated that MALAT1 shRNA suppressed the progression of RCC. This investigation further revealed the mechanism of RCC development and provided a new potential target for the clinical treatment of RCC.

\section{Acknowledgements}

Not applicable.

\section{Funding}

No funding was received.

\section{Availability of data and materials}

The datasets used during the present study are available from the corresponding author upon reasonable request.

\section{Authors' contributions}

ZL, ZM and XX conceived, designed the study and performed the experiments. XX wrote the paper and edited the manuscript. All authors read and approved the manuscript and agree to be accountable for all aspects of the research in ensuring that the accuracy or integrity of any part of the work are appropriately investigated and resolved.

\section{Ethics approval and consent to participate}

All experimental protocols were approved by the Ethics Committee of Tai'an City Central Hospital.

\section{Patient consent for publication}

Not applicable.

\section{Competing interests}

The authors state that they have no competing interests. 


\section{References}

1. Siegel R, Ma J, Zou Z and Jemal A: Cancer statistics, 2014. CA Cancer J Clin 64: 9-29, 2014

2. Saini S, Yamamura S, Majid S, Shahryari V, Hirata H, Tanaka Y and Dahiya R: MicroRNA-708 induces apoptosis and suppresses tumorigenicity in renal cancer cells. Cancer Res 71: 6208-6219, 2011.

3. Yu ZH, Zhang Q, Wang YD, Chen J, Jiang ZM, Shi M, Guo X, Qin J, Cui GH, Cai ZM, et al: Overexpression of cyclooxygenase-1 correlates with poor prognosis in renal cell carcinoma. Asian Pac J Cancer Prev 14: 3729-3734, 2013.

4. Bertone P, Stolc V, Royce TE, Rozowsky JS, Urban AE, Zhu X, Rinn JL, Tongprasit W, Samanta M, Weissman S, et al: Global identification of human transcribed sequences with genome tiling arrays. Science 306: 2242-2246, 2004.

5. Djebali S, Davis CA, Merkel A, Dobin A, Lassmann T, Mortazavi A, Tanzer A, Lagarde J, Lin W, Schlesinger F, et al Landscape of transcription in human cells. Nature 489: 101-108, 2012.

6. Friedman RC, Farh KK, Burge CB and Bartel DP: Most mammalian mRNAs are conserved targets of microRNAs. Genome Res 19: 92-105, 2009

7. Song SJ and Pandolfi PP: miR-22 in tumorigenesis. Cell cycle 13 : $11-12,2014$

8. Chen B, Tang H, Liu X, Liu P, Yang L, Xie X, Ye F, Song C, $\mathrm{Xie} X$ and Wei W: miR-22 as a prognostic factor targets glucose transporter protein type 1 in breast cancer. Cancer Lett 356: 410-417, 2015

9. Jafarzadeh-Samani Z, Sohrabi S, Shirmohammadi K Effatpanah H, Yadegarazari R and Saidijam M: Evaluation of miR-22 and miR-20a as diagnostic biomarkers for gastric cancer. Chin Clin Oncol 6: 16, 2017.

10. Zhang H, Tang J, Li C, Kong J, Wang J, Wu Y, Xu E and Lai M: MiR-22 regulates 5-FU sensitivity by inhibiting autophagy and promoting apoptosis in colorectal cancer cells. Cancer Lett 356 781-790, 2015

11. Yang C, Ning S, Li Z, Qin X and Xu W: miR-22 is down-regulated in esophageal squamous cell carcinoma and inhibits cell migration and invasion. Cancer Cell Int 14: 138, 2014.

12. Zuo QF, Cao LY, Yu T, Gong L, Wang LN, Zhao YL, Xiao B and Zou QM: MicroRNA-22 inhibits tumor growth and metastasis in gastric cancer by directly targeting MMP14 and Snail. Cell Death Dis 6: e2000, 2015.

13. Fan W, Huang J, Xiao H and Liang Z: MicroRNA-22 is downregulated in clear cell renal cell carcinoma, and inhibits cell growth, migration and invasion by targeting PTEN. Mol Med Rep 13: 4800-4806, 2016.

14. Orom UA, Derrien T, Beringer M, Gumireddy K, Gardini A Bussotti G, Lai F, Zytnicki M, Notredame C, Huang Q, et al: Long noncoding RNAs with enhancer-like function in human cells. Cell 143: 46-58, 2010 .

15. Szymanski M, Barciszewska MZ, Erdmann VA and Barciszewski J: A new frontier for molecular medicine: noncoding RNAs. Biochim Biophys Acta 1756: 65-75, 2005.

16. Han Y, Liu Y, Gui Y and Cai Z: Long intergenic non-coding RNA TUG1 is overexpressed in urothelial carcinoma of the bladder. J Surg Oncol 107: 555-559, 2013.

17. Gupta RA, Shah N, Wang KC, Kim J, Horlings HM, Wong DJ, Tsai MC, Hung T, Argani P, Rinn JL, et al: Long non-coding RNA HOTAIR reprograms chromatin state to promote cancer metastasis. Nature 464: 1071-1076, 2010.

18. Xie HW, Wu QQ, Zhu B, Chen FJ, Ji L, Li SQ, Wang CM, Tong YS, Tuo L, Wu M, et al: Long noncoding RNA SPRY4-IT1 is upregulated in esophageal squamous cell carcinoma and associated with poor prognosis. Tumour Biol 35: 7743-7754, 2014.

19. Hirata H, Hinoda Y, Shahryari V, Deng G, Nakajima K, Tabatabai ZL, Ishii N and Dahiya R: Long Noncoding RNA MALAT1 promotes aggressive renal cell carcinoma through Ezh2 and Interacts with miR-205. Cancer Res 75: 1322-1331, 2015

20. Tian $\mathrm{X}$ and $\mathrm{Xu}$ G: Clinical value of lncRNA MALAT1 as a prognostic marker in human cancer: Systematic review and meta-analysis. BMJ Open 5: e008653, 2015.

21. Luan W, Li L, Shi Y, Bu X, Xia Y, Wang J, Djangmah HS, Liu X, You $\mathrm{Y}$ and Xu B: Long non-coding RNA MALAT1 acts as a competing endogenous RNA to promote malignant melanoma growth and metastasis by sponging miR-22. Oncotarget 7 : $63901-63912,2016$
22. Zhang H, Chen Z, Wang X, Huang Z, He Z and Chen Y: Long non-coding RNA: A new player in cancer. J Hematol Oncol 6: 37, 2013.

23. Lu MH, Tang B, Zeng S, Hu CJ, Xie R, Wu YY, Wang SM He FT and Yang SM: Long noncoding RNA BC032469, a novel competing endogenous RNA, upregulates hTERT expression by sponging miR-1207-5p and promotes proliferation in gastric cancer. Oncogene 35: 3524-3534, 2016.

24. Yuan JH, Yang F, Wang F, Ma JZ, Guo YJ, Tao QF, Liu F, Pan W, Wang TT, Zhou CC, et al: A long noncoding RNA activated by TGF- $\beta$ promotes the invasion-metastasis cascade in hepatocellular carcinoma. Cancer Cell 25: 666-681, 2014.

25. Wei Y and Niu B: Role of MALAT1 as a prognostic factor for survival in various cancers: A systematic review of the literature with meta-analysis. Dis Markers 2015: 164635, 2015.

26. Gutschner T, Hammerle M and Diederichs S: MALAT1 - a paradigm for long noncoding RNA function in cancer. J Mol Med 91: 791-801, 2013.

27. Yu F, Lu Z, Cai J, Huang K, Chen B, Li G, Dong P and Zheng J: MALAT1 functions as a competing endogenous RNA to mediate Racl expression by sequestering miR-101b in liver fibrosis. Cell Cycle 14: 3885-3896, 2015.

28. Lu H, He Y, Lin L, Qi Z, Ma L, Li L and Su Y: Long non-coding RNA MALAT1 modulates radiosensitivity of HR-HPV+ cervical cancer via sponging miR-145. Tumour Biol 37: 1683-1691, 2016.

29. Xiao H, Tang K, Liu P, Chen K, Hu J, Zeng J, Xiao W, Yu G, Yao W, Zhou H, et al: LncRNA MALAT1 functions as a competing endogenous RNA to regulate ZEB2 expression by sponging miR-200s in clear cell kidney carcinoma. Oncotarget 6 : 38005-38015, 2015.

30. Xiong J: Emerging roles of microRNA-22 in human disease and normal physiology. Curr Mol Med 12: 247-258, 2012.

31. Budd WT, Seashols-Williams SJ, Clark GC, Weaver D, Calvert V, Petricoin E, Dragoescu EA, O'Hanlon K and Zehner ZE: Dual action of miR-125b as a tumor suppressor and OncomiR-22 promotes prostate cancer tumorigenesis. PLoS One 10: e0142373, 2015.

32. Palacios F, Abreu C, Prieto D, Morande P, Ruiz S, Fernández-Calero T, Naya H, Libisch G, Robello C, Landoni AI, et al: Activation of the PI3K/AKT pathway by microRNA-22 results in CLL B-cell proliferation. Leukemia 29: 115-125, 2015.

33. Zhang S, Zhang D, Yi C, Wang Y, Wang $\mathrm{H}$ and Wang J: MicroRNA-22 functions as a tumor suppressor by targeting SIRT1 in renal cell carcinoma. Oncol Rep 35: 559-567, 2016.

34. Tang Y, Jin X, Xiang Y, Chen Y, Shen CX, Zhang YC and Li YG: The lncRNA MALAT1 protects the endothelium against ox-LDL-induced dysfunction via upregulating the expression of the miR-22-3p target genes CXCR2 and AKT. FEBS Lett 589: 3189-3196, 2015

35. Wang D, Chen J, Chen H, Duan Z, Xu Q, Wei M, Wang L and Zhong M: Leptin regulates proliferation and apoptosis of colorectal carcinoma through PI3K/Akt/mTOR signalling pathway. J Biosci 37: 91-101, 2012.

36. Wang C, Mao ZP, Wang L, Wu GH, Zhang FH, Wang DY and Shi JL: Long non-coding RNA MALAT1 promotes cholangiocarcinoma cell proliferation and invasion by activating PI3K/Akt pathway. Neoplasma 64: 725-731, 2017.

37. Dong Y, Liang G, Yuan B, Yang C, Gao R and Zhou X: MALAT1 promotes the proliferation and metastasis of osteosarcoma cells by activating the PI3K/Akt pathway. Tumour Biol 36: 1477-1486, 2015.

38. Zhou X, Liu S, Cai G, Kong L, Zhang T, Ren Y, Wu Y, Mei M, Zhang L and Wang X: Long non coding RNA MALAT1 promotes tumor growth and metastasis by inducing epithelial-mesenchymal transition in oral squamous cell carcinoma. Sci Rep 5: 15972 , 2015.

39. Ji Q, Zhang L, Liu X, Zhou L, Wang W, Han Z, Sui H, Tang Y, Wang Y, Liu N, et al: Long non-coding RNA MALAT1 promotes tumour growth and metastasis in colorectal cancer through binding to SFPQ and releasing oncogene PTBP2 from SFPQ/PTBP2 complex. Br J Cancer 111: 736-748, 2014 